FERMILAB-TM-2071

\title{
Comparison of the Total Cross Sections Measurements of CDF and E811
}

\author{
M. Albrow et al. \\ Fermi National Accelerator Laboratory \\ P.O. Box 500, Batavia, Illinois 60510
}

February 1999 


\section{Disclaimer}

This report was prepared as an account of work sponsored by an agency of the United States Government. Neither the United States Government nor any agency thereof, nor any of their employees, makes any warranty, expressed or implied, or assumes any legal liability or responsibility for the accuracy, completeness, or usefulness of any information, apparatus, product, or process disclosed, or represents that its use would not infringe privately owned rights. Reference herein to any specific commercial product, process, or service by trade name, trademark, manufacturer, or otherwise, does not necessarily constitute or imply its endorsement, recommendation, or favoring by the United States Government or any agency thereof. The views and opinions of authors expressed herein do not necessarily state or reflect those of the United States Government or any agency thereof.

\section{Distribution}

Approved for public release; further dissemination unlimited.

\section{Copyright Notification}

This manuscript has been authored by Universities Research Association, Inc. under contract No. DE-AC02-76CHO3000 with the U.S. Department of Energy. The United States Government and the publisher, by accepting the article for publication, acknowledges that the United States Government retains a nonexclusive, paid-up, irrevocable, worldwide license to publish or reproduce the published form of this manuscript, or allow others to do so, for United States Government Purposes. 
CDF/PUB/CDF/PUBLIC/4844/

Version 2.0

February 26, 1999

\title{
Comparison of the Total Cross Sections Measurements of CDF and E811
}

\author{
M. Albrow and A. Beretvas, Fermi National Accelerator Laboratory \\ L. Nodulman, Argonne National Laboratory \\ P. Giromini, Laboratori Nazionali di Frascati
}

The total cross section at $\sqrt{s}=1.8 \mathrm{TeV}$ has been measured by three groups (CDF, E710, and E811). We think that CDF should quote results based only on our own measurement. We also indicate how to compare cross sections measured by both CDF and D0.

\section{INTRODUCTION}

The CDF cross section result is $\left(1+\rho^{2}\right) \sigma_{\text {total }}=81.83 \pm 2.29 \mathrm{mb}$ [1]. Using $\rho=0.14$ the CDF result is $80.26 \pm 2.25 \mathrm{mb}$. The other two measurements are FNAL-E710 $\sigma_{\text {total }}=72.81 \pm 3.1 \mathrm{mb}[2]$ and a new measurement FNAL-E811 $\sigma_{\text {total }}=71.71 \pm 2.02 \mathrm{mb}[3]$. Indeed, one finds the following statement in the E811 paper, "Our result is in good agreement with that of E710, and differs by about 2.8 standard deviations from that of $\mathrm{CDF}$; the confidence level that all 3 results are compatible is only $1.6 \%$."

\section{EXPERIMENTAL METHOD}

All three experiments use the same method which is the determination of the total cross using the luminosity independent method.

$$
\left(1+\rho^{2}\right) \sigma_{\text {total }}=16 \pi(\hbar c)^{2} \cdot \frac{\mathrm{A}}{\mathrm{N}_{\mathrm{el}}+\mathrm{N}_{\text {inel }}}=19.572 \frac{\mathrm{mb}}{\mathrm{GeV}^{2}} \cdot \frac{\mathrm{A}}{\mathrm{N}_{\mathrm{el}}+\mathrm{N}_{\text {inel }}}
$$

where $\mathrm{A}=\mathrm{dN} \mathrm{Ne}_{\mathrm{el}} /\left.\mathrm{dt}\right|_{t=0}, \mathrm{~N}_{\mathrm{el}}$ is the elastic cross section and $\mathrm{N}_{\text {inel }}$ is the inelastic cross section. The intercept $\mathrm{A}$ is determined by extrapolating the elastic cross to $\mathrm{t}=0$ and the functional form is: 


$$
\mathrm{N}_{\mathrm{el}}=\mathrm{A} / \mathrm{b}
$$

where $\mathrm{b}$ is the slope (see Fig. 9 of [4]) on a semi log plot of $\mathrm{d} \sigma / \mathrm{d} t$ versus $t$. As E811 has data (reported in their paper) only over the range $0.0045<-t<0.036 \mathrm{GeV}^{2}$ they use the a combination of the slope from $\mathrm{CDF} \mathrm{b}=16.98 \pm 0.24(\mathrm{GeV} / c)^{-2}$ and from $\mathrm{E} 710 \mathrm{~b}=16.99 \pm 0.47(\mathrm{GeV} / c)^{-2}$. The CDF measurement is over the range $0.04<-t<0.29 \mathrm{GeV}^{2}$. The inelastic cross can be written as:

$$
\mathrm{N}_{\text {inel }}=\mathrm{N}_{\mathrm{i}}+\mathrm{N}_{\mathrm{sd}}
$$

where $\mathrm{N}_{\mathrm{i}}$ is the number of events with a two-sided coincidence in either the BBC or forward telescope, and $\mathrm{N}_{\mathrm{SD}}$ is the number of events with a single $\bar{p}$ detected in the forward magnetic spectrometer coincident with hits in the opposite side BBC or forward telescope. There is good agreement between the single diffractive measured by CDF $9.46 \pm 0.44 \mathrm{mb}[5]$ and E710 $8.1 \pm 1.7 \mathrm{mb}$ [6], $11.7 \pm 2.3 \mathrm{mb}$ [7]. Although the single diffractive number is not directly used by E811, as a check they use our measured number (world average) and find a change of only $0.3 \mathrm{mb}$ in their answer for the total cross section.

\section{REASONS WHY WE PREFER TO USE THE CDF MEASUREMENT ONLY}

\section{A. CDF}

The CDF results are documented in great detail in 3 papers [4], [5] and [1].

The same method that is used for our analysis at $\sqrt{s}=1.8 \mathrm{TeV}$ is also used at $\sqrt{s}=0.546 \mathrm{TeV}$. Our result for the total cross section at $\sqrt{s}=0.546$ is $61.26 \pm 0.93 \mathrm{mb}$ in good agreement with the UA4 result of $61.9 \pm 1.5 \mathrm{mb}$ [8]. We note that the CDF measurement has a significantly smaller error than UA4 due to better accuracy in measuring inelastic and diffractive rates.

Our experiment covered the pseudorapidity range $3.2<\eta<6.7$ with several telescopes of scintillation counters. Since more than $90 \%$ of the events fire all telescopes we had a good understanding of the trigger efficiency. In addition, our experiment allows one to examine the inelastic events in great detail. A VTPC (Vertex Time Projection Chamber) allows one to eliminate background that is in the tails of the distribution 
(see Fig.3 [1]). The forward telescopes allow the shape of the beam gas background to be determined. The background issues are very important as the experiment is done at low luminosity $\left(1.9 \times 10^{26} \mathrm{~cm}^{-2} \mathrm{sec}^{-1}\right)$. Note if no vertex analysis flow analysis is done, as is the case for E811 (see table III) we would get a total cross section of $74.0 \mathrm{mb}$.

Many aspects of our measurement are understood with a detailed simulation which reproduces the measured angular and multiplicity distributions ; we use the simulation to account for $1.3 \%$ of the inelastic events lost by our trigger. In particular the diffractive cross section is measured directly without any extrapolation. The accuracy of our elastic spectrometer is comparable to the UA4 experiment. In principle one small angle detector on each side of the interaction region is enough to measure the elastic cross section. On the west side (outgoing $\bar{p}$ ) we have three detectors $\mathrm{S} 3, \mathrm{~S} 2$ and $\mathrm{S} 1$. On the east side (outgoing $p$ ) we have two detectors S6 and S7. Each spectrometer detector comprised a drift chamber and a silicon detector sandwiched by two scintillation counters.

This feature allowed us to measure all systematic uncertainties which affect this type of experiment:

- detector efficiencies, including the measurement of events lost because of overlapping beam splashes

- focal lengths of each telescope

- geometrical acceptance

- shape of the interaction region and beam angular divergence

- effect of nuclear interactions in the detectors

A nice example of this is the spectrometer $t$ acceptance given in Fig 23 of [4]. The spectrometer detectors were surveyed to an accuracy of $0.1 \mathrm{~mm}$. However, the situation changes during the actual run. We were able to confirm our understanding of the position of the beam by making measurements and a detailed simulation of the magnetic lattice. A very important part of our measurement is the detailed list of systematic errors given in table VI [4]. 


\section{B. More Details on the CDF Errors}

The relevant numbers for deriving the total cross section are $\mathrm{N}_{\mathrm{i}}=208890 \pm 2558(1.2 \%), \mathrm{N}_{\mathrm{sd}}=32092 \pm$ $1503(4.7 \%), \mathrm{A}=1336532 \pm 40943 \mathrm{GeV}^{-2}(3.1 \%)$ and $\mathrm{b}=16.98 \pm 0.025 \mathrm{GeV}^{-2}$ (1.5\%). In addition we need to know the covariance $(\mathrm{A}, \mathrm{b})=0.93\left(\mathrm{~N}_{\mathrm{el}}=78691 \pm 1463(1.9 \%)\right)$. The equation for the error is:

$$
\begin{gathered}
\left(1+\rho^{2}\right)^{2}\left(\Delta \sigma_{\text {total }}\right)^{2}=\left(\frac{\mathrm{d} \sigma_{\text {total }}}{\mathrm{dN} N_{\mathrm{i}}}\right)^{2}\left(\Delta \mathrm{N}_{\mathrm{i}}\right)^{2}+\left(\frac{\mathrm{d} \sigma_{\text {total }}}{\mathrm{dN} N_{\mathrm{sd}}}\right)^{2}\left(\Delta \mathrm{N}_{\mathrm{sd}}\right)^{2}+\left(\frac{\mathrm{d} \sigma_{\text {total }}}{\mathrm{d} A}\right)^{2}(\Delta A)^{2}+ \\
\left(\frac{\mathrm{d} \sigma_{\text {total }}}{\mathrm{d} b}\right)^{2}(\Delta b)^{2}+2 \cdot \operatorname{cov}(A, b) \frac{\mathrm{d} \sigma_{\text {total }}}{\mathrm{d} A} \frac{\mathrm{d} \sigma_{\text {total }}}{\mathrm{d} b} \Delta A \Delta b
\end{gathered}
$$

It should be mentioned that this numerically becomes

$$
\left(1+\rho^{2}\right)^{2}\left(\Delta \sigma_{\text {total }}\right)^{2}=0.429+0.148+3.571+0.088+1.042 .=5.278
$$

The error on $\sigma_{\text {total }}$ is $2.3 \mathrm{mb}$. Note that we have estimated the systematic error on A to be $0.48 \%$, and the systematic error on $\mathrm{N}_{\mathrm{el}}$ to be $0.54 \%$.

\section{The E811 experiment}

The experiment has lots of statistics. The number of elastic events is $\approx 28 \mathrm{~K}$, while the corresponding $\mathrm{CDF}$ number is $\approx 9 \mathrm{~K}$. The number of inelastic events is $\approx 1350 \mathrm{~K}$ while the corresponding CDF number is $\approx 14 \mathrm{~K}$. They made 10 different runs which are in very good agreement (high $73.43 \pm 1.22 \mathrm{mb}$ and low 69.93 $\pm 3.55 \mathrm{mb}) \quad[9]$.

Elastic events are detected by using scintillating fiber high resolution detectors. These detectors have particle resolution of $38 \mu \mathrm{m}$ and detection efficiency $>97 \%$. Unfortunately, they have only one elastic detector per side.

A set of 3 annular scintillation counters called L and a similar set of 3 called $\mathrm{R}$ are used to measure the inelastic cross section. These counters cover the range $5.2<\eta<6.5$, smaller than CDF. E811 has no wire chambers to distinguish between real $p \bar{p}$ interactions and background. 


\section{Details of the error calculation for E811}

Unfortunately the documentation consist only of one short Letter [3] and some transparencies [9]. The number of inelastic events is given by the formula

$$
\mathrm{N}_{\text {inel }}=\mathrm{N}_{\mathrm{LR}}+\mathrm{N}_{\mathrm{L}+\mathrm{R}}+\mathrm{N}_{\mathrm{L}+\mathrm{R}}(\text { losses })=(1350.3 \pm 6.4+434.8 \pm 56.7+14.9 \pm 8.1) \mathrm{K}=(1800+/-57.2) \mathrm{K}
$$

where $\mathrm{N}_{\mathrm{LR}}$ is the number of $\mathrm{LR}$ coincidences, where $\mathrm{N}_{\mathrm{L}+\mathrm{R}}$ is the single arm rate after background subtraction, and $\mathrm{N}_{\mathrm{L}+\mathrm{R}}$ is a small correction term because not all of $\eta$ is covered. Since they do not have wire chambers, they don't investigate background in the LR rate. Probably not a problem, but the counter efficiencies are not discussed.

To quote from the E811 publication "As in E-710, the single arm data used for obtaining $\mathrm{N}_{\text {inel }}$ has a significant $(\approx 93 \%)$ background caused by, for example beam-gas interactions." The background levels are studied by running with missing bunches. Because of space limitations there is essentially no discussion of the systematic errors associated with this missing bunch study. However, they are included in the transparencies from the seminar given by Carlos Avila [9], and evaluated to be about 13\%. UA4 had chamber telescopes to distinguish beam-beam and beam-gas interactions and they measured the single arm contribution with a 9\% error. The missing bunch procedure does not address all background issues. We think that indeed the $\mathrm{N}_{\mathrm{L}+\mathrm{R}}$ is high, because we also have counters that have the same rapidity coverage as those used by E811. We measure that " $\mathrm{N}_{\mathrm{L}+\mathrm{R}} / \mathrm{N}_{\mathrm{LR}}$ " is $21 \%$ while the number from $\mathrm{E} 811$ is $32 \%$.

We are also told that $\mathrm{A}=(8628 \pm 60) \mathrm{K}\left(\mathrm{GeV}^{-2}\right)$ which corresponds to a $0.7 \%$ error and that $\mathrm{N}_{\mathrm{el}}=$ $(508.1 \pm 3.5) \mathrm{K}$ which corresponds to a $0.7 \%$ error. For both $\mathrm{A}$ and $\mathrm{N}_{\mathrm{el}}$ there is no table of systematic

errors corresponding to vertex cut, TOF losses, Backgrounds, Magnetic Lattice, x scale, tilt angle, nuclear interactions, and beam momentum. We are just told that the error is predominantly statistical.

\section{HOW LUMINOSITY IS MEASURED AT CDF}

The CDF luminosity is measured using the beam-beam counters (BBC). The beam-beam counters consist of two planes of scintillation counters covering the angular range $0.32^{\mathrm{deg}}$ to $4.47^{\mathrm{deg}}$ in both the proton and 
antiproton directions $(3.24<\eta<5.90)$. The BBC cross section is related to the total cross section

$$
\sigma_{\mathrm{BBC}}=\sigma_{\text {total }} \frac{\mathrm{N}_{\mathrm{BBC}}}{\mathrm{N}_{\mathrm{inel}}+\mathrm{N}_{\mathrm{el}}}
$$

This we can rewrite as

$$
\sigma_{\mathrm{BBC}}=\sigma_{\text {total }} \frac{\left(\frac{\mathrm{N}_{\mathrm{BBC}}}{\mathrm{N}_{\mathrm{i}}}\right) \mathrm{N}_{\mathrm{i}}}{\mathrm{N}_{\mathrm{i}}+\mathrm{N}_{\mathrm{sd}}+\mathrm{N}_{\mathrm{el}}}
$$

The quantity $\mathrm{N}_{\mathrm{i}}$ is a superset of $\mathrm{N}_{\mathrm{BBC}}$ and includes a Monte Carlo acceptance correction of $1.2 \%$. We find 98.7\% of $\mathrm{N}_{\mathrm{i}}$ triggered events are BBC triggered events. Therefore $\mathrm{N}_{\mathrm{BBC}} / \mathrm{N}_{\mathrm{i}}=0.987 / 1.012=0.975$.

$$
\sigma_{\mathrm{BBC}}=0.975 \sigma_{\mathrm{i}}=0.975\left(\sigma_{\text {inel }}-\frac{32092}{37782} \cdot \sigma_{\mathrm{sd}}\right)=0.975\left(60.33 \cdot 1.0028-\frac{32092}{37782} \cdot 9.46\right)
$$

The beam-beam cross section is $51.15 \pm 1.60 \mathrm{mb}$ [10]. The luminosity depends inversely on the beam-beam cross section:

$$
\mathrm{L}=\frac{\mathrm{N}_{\mathrm{BBC}}}{\sigma_{\mathrm{BBC}}}
$$

where $\mathrm{N}_{\mathrm{BBC}}$ is the number of $\mathrm{BBC}$ interactions during the time $\mathrm{CDF}$ is "live". The number of $\mathrm{BBC}$ interactions is given in terms of $\mathrm{N}_{\mathrm{BC}}$ (number of beam crossings), $\mathrm{N}_{\mathrm{EW}}$ (number of crossings that contain a coincidence), $\mathrm{N}_{\mathrm{E}}$ (number of crossings that contain a hit in the east beam counters), and $\mathrm{N}_{\mathrm{W}}$ (number of crossings that contain a hit in the west beam counter). The details for this calculation are given in CDF $4721[11]$.

The issue of "Standardization of CDF and D0 reported Luminosities" for reporting during Run I is discussed in a Fermilab-TM [12]. The luminosity reported to the accelerator division was based on beambeam cross section of $49.9 \mathrm{mb}$. This is obtained by using a weighted average of the CDF and E710 (see Table 2 of [13]) inelastic cross section $(58.9 \pm 1.2 \mathrm{mb}))$ and then using:

$$
\sigma_{\mathrm{BBC}}(\operatorname{avg})=\sigma_{\mathrm{BBC}}(\mathrm{CDF}) \frac{\sigma_{\text {inel }}(\mathrm{avg})}{\sigma_{\text {inel }}(\mathrm{CDF})}
$$

This yields $\sigma_{\mathrm{BBC}}(\mathrm{avg})=49.9 \mathrm{mb}$ which differs from the CDF value by $2.4 \%$. In terms of numbers quoted in papers (rather than reported to the accelerator division) we expect that the luminosity reported by D0 would be $2.4 \%$ high and the cross sections would be $2.4 \%$ low. If we use equ. 4.3 rather than 4.5 we find 
the luminosity reported by D0 is $2.7 \%$ high and the cross section would be $2.7 \%$ low. In publications on the measurement of the $W$ and $Z$ cross section we have just quoted the measured numbers [10]. This has been reasonable as the error on our luminosity for Run Ia is 3.6\%. In addition the statistical error on the $W$ measurement was $3.2 \%$.

\section{HOW LUMINOSITY IS MEASURED AT DO}

D0 uses two hodoscope scintillator arrays to measure it's instantaneous luminosity. The arrays have nearly complete coverage for $2.2<\eta<3.9$ [13]. These detectors are more than $99 \%$ efficient in detecting non-diffractive inelastic collisions. The equivalent beam-beam cross section is

$$
\sigma_{\mathrm{L} 0}=\epsilon_{\mathrm{L} 0}\left(\epsilon_{\mathrm{HC}} \sigma_{\mathrm{HC}}+\epsilon_{\mathrm{SD}} \sigma_{\mathrm{SD}}+\epsilon_{\mathrm{DD}} \sigma_{\mathrm{DD}}\right)
$$

where $\sigma_{\mathrm{HC}}$ is the hard component of the inelastic cross section, $\sigma_{\mathrm{SD}}$ is the single diffractive component, and $\sigma_{\mathrm{DD}}$ is the double diffractive component. The acceptances are determined by two different Monte Carlo programs. These programs are DTUJET and MBR (the numbers are in tables 5 and 6 of Ref [13]). The average acceptance is used $\left(\epsilon_{\mathrm{HC}}=(97.1 \pm 0.5(\right.$ stat $) \pm 1.9($ syst $)) \%, \epsilon_{\mathrm{SD}}=(15.1 \pm 0.8$ (stat) \pm 5.4 (syst $\left.)\right) \%$, $\epsilon_{\mathrm{DD}}=(71.6 \pm 1.1$ (stat) \pm 3.1 (syst) $\left.) \%\right)$. They use a weighted average of the CDF and E710 numbers for the single diffractive cross section $(9.54 \pm 0.43 \mathrm{mb})$. The double diffractive number is obtained from

$$
\sigma_{\mathrm{DD}}=\frac{\left(\sigma_{\mathrm{SD}}\right)^{2}}{4 \sigma_{\mathrm{el}}}
$$

The corresponding numbers from CDF and E710 are $1.14 \pm 0.12 \mathrm{mb}$ and $2.1 \pm 0.8 \mathrm{mb}$ resulting in an average number of $1.15 \pm 0.17 \mathrm{mb}$. The hard core component is determined by using

$$
\sigma_{\mathrm{HC}}=\sigma_{\mathrm{inel}}-\sigma_{\mathrm{SD}}-\sigma_{\mathrm{DD}}
$$

Using the weighted averages from CDF and E710 they find $\sigma_{\mathrm{HC}}=48.25 \pm 2.23 \mathrm{mb}$. Their final number using $\epsilon_{\mathrm{L} 0}=0.95$ is $\sigma_{\mathrm{L} 0}=46.7 \pm 2.5 \mathrm{mb}$. If only the CDF numbers are used than $\sigma_{\mathrm{L} 0}=48.0 \mathrm{mb}$. Using the CDF numbers would make the integrated luminosity lower by $2.9 \%$, and would increase measured cross sections by the same amount. For run Ib the efficiency D0 finds is $\epsilon_{\mathrm{L} 0}=0.907 \pm 0.017$ [14]. The new number is 
the result of an upgrade in electronics and small corrections for halo and multiple single diffraction. This reduces the beam-beam cross section to $44.53 \pm 2.37 \mathrm{mb}$. The situation is the same in run Ib as run Ia with respect to using the $\mathrm{CDF}$ numbers as only the efficiency $\epsilon_{\mathrm{L} 0}$ has changed.

\section{CONCLUSIONS}

We have pointed out why we prefer to use our own measurement for the luminosity; and how best to compare the measurements of $\mathrm{CDF}$ and D0. We understand that D0 will adopt the new world average for the inelastic cross section (CDF, E710 and E811). Thus the D0 numbers will have $6.1 \%$ more luminosity and a cross section that is $6.1 \%$ lower than CDF. We and D0 have agreed that $W$ and $Z$ cross sections, where the difference is significant, should be clearly labeled, and that combined numbers should clearly call out that they are renormalizing one or the other, according to the speaker's preference.

It is not obvious what we will do in run II when the machine is at $\sqrt{s}=2$. TeV. CDF will have a new system for monitoring luminosity consisting of low mass Cherenkov detectors [15]. We think that giving cross sections relative to that of the $W$ may be a way to avoid many of the difficulties in a detailed understanding of how luminosity is measured at CDF and D0. We also would recommend that the accelerator division should again do Van Der Meer scans to measure the luminosity. Silicon detectors and improved beam steering offer at least some hope that scans may do better than in the past.

[1] F. Abe et al., Phys. Rev. D 50, 5550 (1994).

[2] N. Amos et al., Phys. Rev. Lett. 68, 2433 (1992).

[3] C. Avila et al., Phys. Lett. B 445, 419 (1999).

[4] F. Abe et al., Phys. Rev. D 50, 5518 (1994).

[5] F. Abe et al., Phys. Rev. D 50, 5535 (1994). 
[6] N. Amos et al., Phys. Lett. B301, 313 (1993).

[7] N. Amos et al., Phys. Lett. B243, 158 (1990).

[8] M. Bozzo et al., Phys. Lett. B147, 392 (1984).

[9] Seminar given by Carlos Avila at Fermilab on Sept. 16, 1998.

[10] F. Abe et al. Phys. Rev. Lett. 76, 3070 (1996).

[11] D. Cronin-Hennessy and A. Beretvas, CDF-4721, Nov. 1998.

[12] J. Bantly, R. Plunkett, S. Pruss and M. Tartaglia, Fermilab-TM-1906, Oct. (1994).

[13] J. Bantly, A. Brandt, R. Partridge, J. Perkins, and D. Puseljic, Fermilab-TM-1930, Mar. (1996).

[14] J. Bantly, J. Krane, D. Owen, R. Partridge and L. Paterno, Fermilab-TM-1995, June (1997).

[15] S. Klimenko et al., CDF-4330, Nov. 1997.

[16] Henry Frisch, CDF-2484, July 1994. 